\title{
Daily Moxifloxacin, Clarithromycin, Minocycline, and Clofazimine in Nonresponsiveness Leprosy Cases to Recommended Treatment Regimen
}

\author{
Francisco Bezerra de Almeida Neto, $\mathrm{MD}^{1,2,3^{*}}$, Rebeca Daniele Buarque Feitosa, $O T^{3}$ and Marqueline \\ Soares da Silva, $R N^{3}$
}

${ }^{1}$ Department of Dermatology, Mauricio de Nassau Recife University Center, Brazil

${ }^{2}$ Department of Tropical Medicine, Federal University of Pernambuco (UFPE), Brazil

${ }^{3}$ Cabo de Santo Agostinho Health Care Hansen's Disease Specialized Center, Cabo de Santo Agostinho, Brazil

*Corresponding author: Francisco Bezerra de Almeida Neto, MD, Department of Dermatology, Mauricio de Nassau Recife University Center; Department of Tropical Medicine, Federal University of Pernambuco (UFPE); Cabo de Santo Agostinho Health Care Hansen's Disease Specialized Center, Cabo de Santo Agostinho, R Jonathas de Vasconcelos, 316, Boa Viagem, Recife, PE, CEP 51021140, Brazil, Tel: +5581988484442

\begin{abstract}
Background: In hyperendemic countries for leprosy, there has been a growing increase in clinical multibacillary "nonresponsiveness" leprosy cases to the fixed-duration treatment recommended by World Health Organization (MDT$\mathrm{MB})$. There are no defined protocols to treat these patients.

Methods: A retrospective, observational case series study was conducted of 4 patients with multibacillary leprosy who presented to a specialized Leprosy health care. Between July 1, 2018, and July 31,2020 , they were treated with moxifloxacin, clarithromycin, minocycline, and clofazimine. A literature review was conducted on the clinical features and treatment of nonresponsiveness leprosy cases to regular MDT-MB.

Results: Four patients ( 3 male; mean [SD] age at treatment, 41 [22.6] years) with nonresponsiveness lepromatous leprosy to regular MDT-MB were successfully treated with daily moxifloxacin, clarithromycin, minocycline and clofazimine for 24 months. There were reduction in the number and size of skin lesions and infiltrations, neural thickening (both visually and by palpation), bacteriological index, and leprosy reactions at 6 to 24 months after initiation of therapy.

Conclusions: This case series suggests that daily moxifloxacin, clarithromycin, minocycline and clofazimine association may hold promise as a treatment for refractory lepromatous leprosy to regular MDT-MB.
\end{abstract}

\section{Keywords}

Leprosy, Lepromatous Leprosy, Drug resistance

\section{Introduction}

Although leprosy is the first infectious disease attributed to a pathogen by Gerard Amauer Hansen, it remains a relevant public health problem in countries considered hyper-endemic for the disease [1].

The main etiologic agents are Mycobacterium leprae and Mycobacterium lepromatosis. The clinical manifestations of the disease are diverse, according to the pattern of genetically predetermined immune response $[2,3]$.

The WHO recommended treatment (MDT-MB-12 doses) has contributed to the reduction of the prevalence coefficient of the disease over the years. However, there is an increase trend of pediatric cases with grade 2 disability, polar lepromatous (LL), and other multibacillary cases. LL or subpolar lepromatous leprosy patients (BL) remain a challenge for specialists who accompany them in specialized care centers [4].

There is no consensus in the literature to assess criteria for a cure in patients after regular MDT-MB. Patients with high initial bacteriological index (BI), who remain without improvement after the completion of treatment, should extend it by an additional 12 doses, according national guidelines in Brazil. This situation is

Citation: Neto FBA, Feitosa RDB, da Silva MS (2020) Daily Moxifloxacin, Clarithromycin, Minocycline, and Clofazimine in Nonresponsiveness Leprosy Cases to Recommended Treatment Regimen. Int J Trop Dis 3:035. doi.org/10.23937/2643-461X/1710035

Accepted: October 05, 2020; Published: October 07, 2020

Copyright: (C) 2020 Neto FBA, et al. This is an open-access article distributed under the terms of the Creative Commons Attribution License, which permits unrestricted use, distribution, and reproduction in any medium, provided the original author and source are credited. 
considered "therapeutic insufficiency". If after 24 doses of regular MDT-MB, patients remain without improvement, these cases are called "therapeutic failure" and drug resistance test (DR) should be performed. There is no recommendation on treatment after the DR test result [5].

Therefore, no well-established, effective cure criteria and treatment protocols exist for nonresponsiveness leprosy cases (NR-Lep). The main ways of assessing the clinical improvement of these patients are the appearance of the lesions at the end of treatment and the decline in $\mathrm{BI}$ in log-units per year, determinate previously by internationally used Ridley's logarithmic scale [6].

Antibody responses to specific Mycobacterium leprae antigens can be evaluated by levels of immunoglobulin $\mathrm{M}$ (IgM) against phenolic glycolipid-1 (PGL-1). The presence of high titles of anti-PGL-1 IgM reflects the total bacterial load in the body, however, test anti-PGL-1 are not widely available in field, being restricted to research institutions $[7,8]$.

In fact, there is no consensus as to what the actual patients' cure means. The established cure happens only because these patients complete the period recommended for treatment with MDT-MB, which is insufficient in several cases of relapse of the disease mainly in lepromatous leprosy [9].

Moxifloxacin, clarithromycin, minocycline, and clofazimine (MCMC) used for the treatment of this case series patients are mentioned in the WHO guidelines, still without retrieved evidence, based on the opinion of experts [10].

\section{Methods}

We conducted a retrospective case series of 4 patients with NR-lep presenting to Municipal Cabo de Santo Agostinho Health Care Hansen's Disease Specialized Center (Cabo de Santo Agostinho - Pernambuco - Brazil) between July 1, 2018, and July 31, 2020. This project was not human participants' research, and the Brazilian Ministry of Health received a report on the clinical situation and treatment of this case series. All patients provided written consent.

Four sites provided the collection of skin smears by an experienced laboratory technician: The two ear lobes, right elbow, and an active lesion, according to the protocol in Brazil. Slides were stained using Ziehl Neelsen Carbol Fushin and scored for $\mathrm{Bl}$, determined for an exclusive Biomedical professional specializing in mycobacteria using international Ridley's logarithmic scale. The laboratory has a regular quality control service both for the technicians and for the field. The Oswaldo Cruz Institute (Fiocruz), Rio de Janeiro - RJ, Brazil performed the molecular biology tests for drug resistance.

\section{Case Series}

Patient 1 was a female patient, 29-years-old, pre-

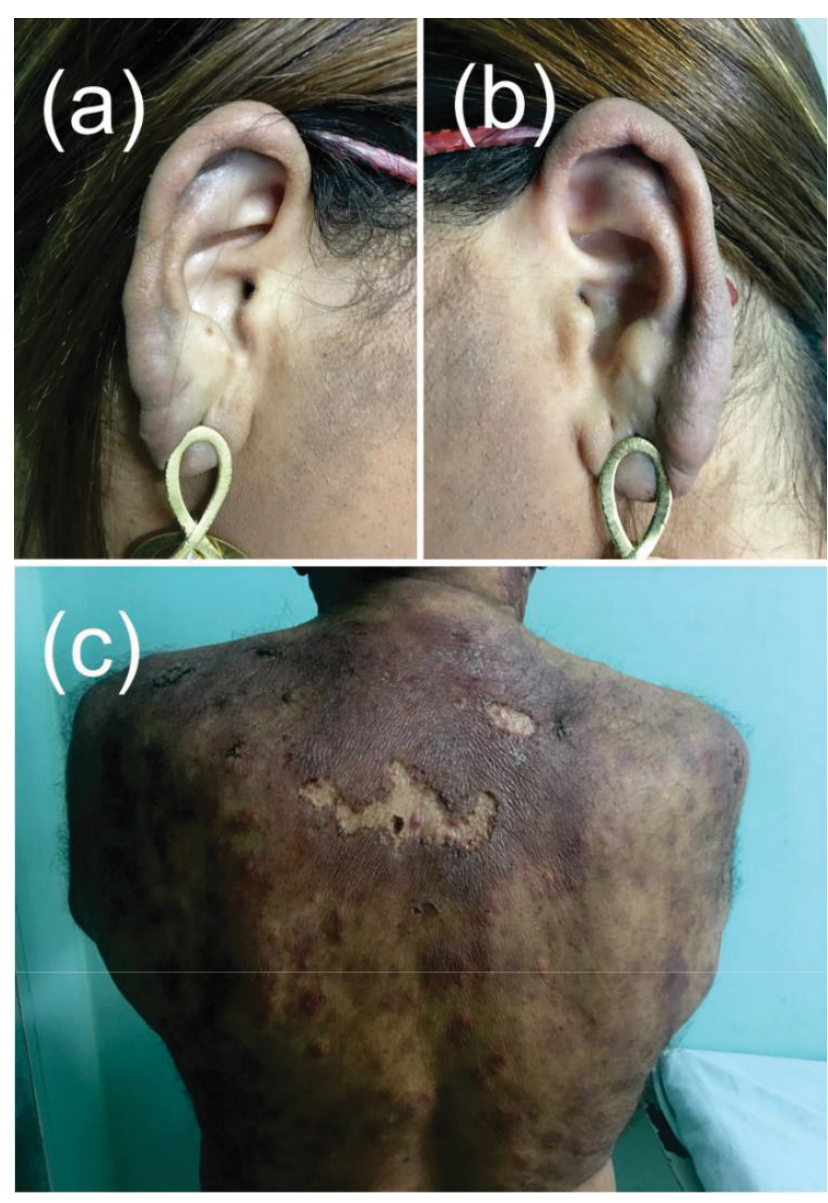

Figure 1: Patient 1 and patient 3 after the $36^{\text {th }}$ of $\mathrm{WHO} /$ MDT/MB (A) Right earlobe patient 1; (B) Left earlobe patient 1 ; (C) Dorsal region patient 3.

senting infiltrations in earlobes and external ears and multiple fibromatous lesions scattered over the skin, predominantly in the abdomen and lower limbs. In addition, there was involvement of the ulnar and posterior tibial nerves. She also had corneal anesthesia on the right. The diagnostic of LL was in May 2013. BI was 5.0. She started MDT-MB. After the first month of treatment, she developed intolerance to dapsone, and ofloxacin $400 \mathrm{mg} /$ day replaced this drug, as recommended by the Brazilian Ministry of Health. After 24 doses of treatment, there was no clinical improvement, showing the lesions with the same initial appearance. There was infiltration of the earlobes and external ears in the same way and BI remained 5.0. Drug resistance test was negative. Additional twelve months extended treatment to $36^{\text {th }}$ month. She concludes it and remained without clinical improvement, with the $\mathrm{BI}=5.0$ (Figure $1 \mathrm{~A}$ and Figure 1B). Subsequent daily MCMC therapy led to improvement of infiltrations 6 months after treatment (Figure $2 \mathrm{~A}$ and Figure $2 \mathrm{~B}$ ). At the 24-month follow-up, the $\mathrm{BI}$ is 0.75 . Patient's skin is free of initial lesions. During the entire follow-up period, this patient developed erythema nodosum leprosum (ENL). The first episode was at the fourth month of treatment and continued after the MCMC treatment.

Patient 2 was male, 30-years-old, diagnosed with LL 


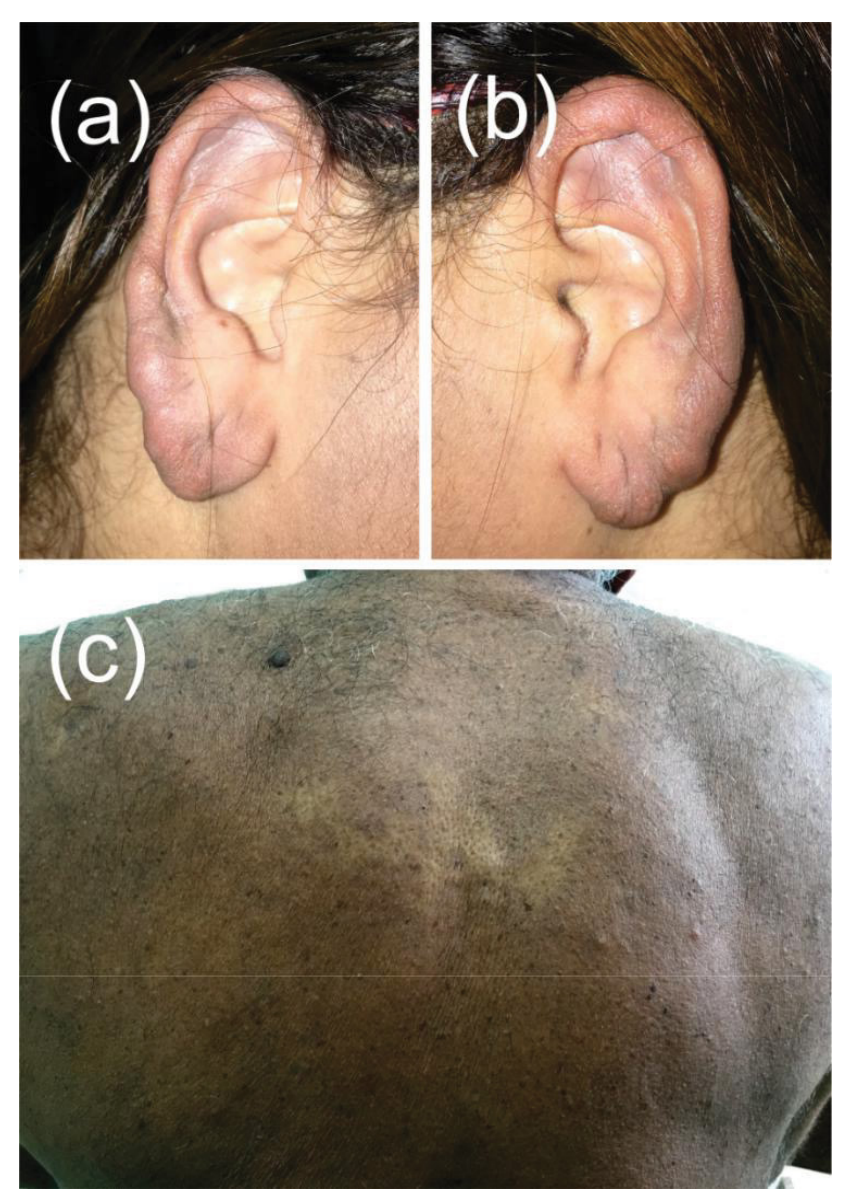

Figure 2: Patient 1 and patient 3 in the $6^{\text {th }}$ month of MCMC treatment $(A)$ Right earlobe patient 1; (B) Left earlobe patient $1 ;(C)$ Dorsal region patient 3.

in April 2013. The dermatological examination did not show many changes, except for a significant thickening of the great auricular nerve (GAN). Due to few skin changes, the patient's diagnosis happened when he developed lesions of ENH. BI was 4.75 . He performed 24 doses of MDT-MB treatment. He developed several episodes of ENL during treatment accompanied by neuritis in the GAN and subsequently in the right ulnar nerve, also denoting the impairment of this last nerve. At the end of the treatment period, the BI was 4.25. The treatment was extended with an additional 12 months with regular MDT, with the $\mathrm{BI}$ at $36^{\text {th }}$ dose $=4.50$. He started the MCMC scheme. After the third month of treatment, he no longer developed leprosy reactions (LR). At the 24-month follow-up, the $\mathrm{BI}=0$.

Patient 3 was a 67-year-old man, diagnosed with BL in May 2013. On examination, he had infiltration in the auricular pavilions, several infiltrated areas along the skin and involvement of the ulnar and posterior tibial nerves, bilaterally. He developed several episodes of type 1 and type 2 leprosy reactions from the third month of treatment, treated in the same way as other patients. The initial BI was 4.50. The treatment for him was with 24 doses of regular MDT-MB. The final BI was 4.25. Drug resistance test was negative. Due to the little decrease in $\mathrm{BI}, 12$ additional doses of regular MDT-
$\mathrm{MB}$ were prescribed. The $\mathrm{BI}$ at the end of the $36^{\text {th }}$ dose remains 4.25 and the patient continued to develop LR (Figure $1 \mathrm{C}$ ). After starting with daily MCMC treatment, $\mathrm{LR}$ ceased at the sixth month of treatment. There was a significant improvement in dermatological lesions (Figure $2 \mathrm{C})$. The final $\mathrm{BI}$ was 0 .

Patient 4 was a 26-year-old man, diagnosed with LL in March 2013. He had infiltration of auricular pavilions and some cutaneous areas, in addition to thickening and palpation pain in ulnar, common fibular and posterior tibial nerves. Initial BI was 4.75. He performed 24 doses of regular MDT-MB. From the first month of treatment, he developed several episodes of ENL with associated neuritis, two of which are more severe and in need of hospital intervention. At the end of treatment, BI was 4.50, and drug resistance tests were negative. The treatment also was extended with an additional 12 months with regular MDT, with the $\mathrm{BI}$ at $36^{\text {th }}$ dose $=4.75$. Subsequent daily MCMC therapy led to improvement of infiltrations 1 year after treatment. He remained with less intense LR throughout the period and continued after the MCMC treatment. The final BI was 0.25 .

All patients were treated for leprosy reactions with corticosteroids (prednisone, dexamethasone, and methylprednisolone), with dosages ranging from 20 to $60 \mathrm{mg} /$ day and chosen according to the intensity and duration of the episode. Besides, all of them also used thalidomide, with different dosages, between 100 to $400 \mathrm{mg} /$ day. Patient 4 required the addition of pentoxifylline, as leprosy reactions were not controlled with the maximum doses of corticosteroids and thalidomide allowed. Amitriptyline ( $50 \mathrm{mg} /$ day to $75 \mathrm{mg} /$ day) was an adjuvant for the control of neuropathic pain in all patients. The use of $100 \mathrm{mg} /$ day of acetylsalicylic acid (ASA) minimized the risk of thromboembolic events. There were no predisposing factors for the occurrence of leprosy reactions (Table 1).

\section{Discussion}

This case series documents the efficacy of 24 months daily MCMC in the treatment of NR-lep in a small cohort.

Skin lesions, neural involvement and leprosy reactions episodes remained unchanged in all patients, after completing 36 doses of MDT-MB treatment. No patient achieved clinical improvement after the end of 36 doses of regular MDT-MB.

BI declined very little and below expectations in all cases. After completing the 24 doses of MDT-MB treatment, all patients underwent drug resistance testing. Due to the negative results, they have same treatment extended by 12 more doses, since the patients did not improve.

The $\mathrm{BI}$ decline after MDT-MB treatment averages 0.5 to 1 unit per year. At the end of the $36^{\text {th }}$ month, the pa- 


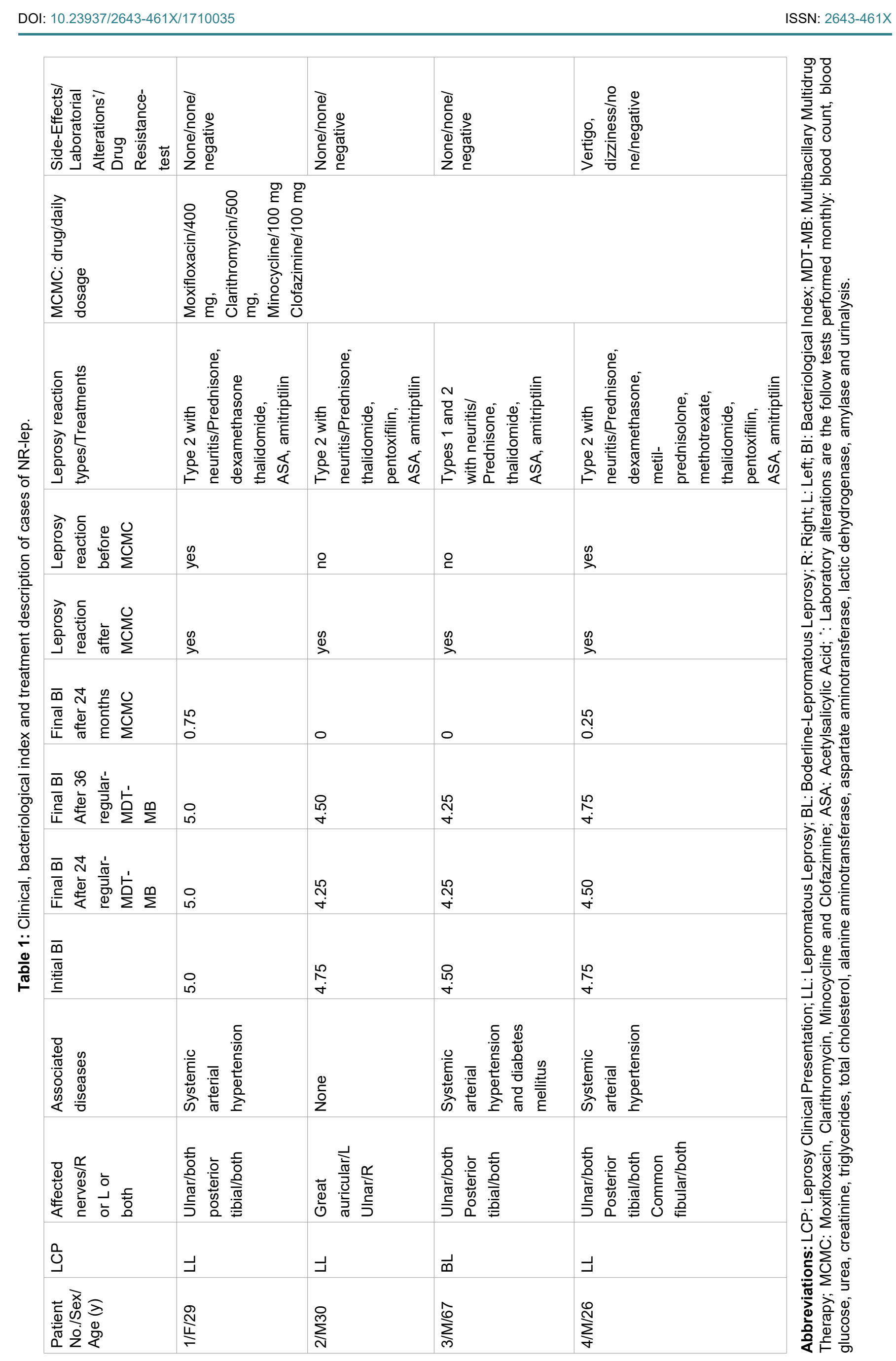


tients also remained without improvement or changes in the $\mathrm{BI}$, except for patient 4 , who increased the $\mathrm{BI}$ to the initial level.

It is important to note that all patients were tested for drug resistance and were negative. For this reason, patients remained on regular treatment with MDT-MB until the $36^{\text {th }}$ dose.

Current tests for drug resistance in leprosy target the genes of the drug resistance determinant region (DRDR) dihydropteroate synthase (Folp1), DNA-directed RNA polymerase subunit beta (RpoB), and DNA gyrase subunit $A(G y r-A)$. However, there are mutations identified in other regions, DRDR's outside, such as bifunctional enzyme riboflavin biosynthesis protein (ribD), DNA-directed RNA polymerase subunit alpha (rpoA), DNA-directed RNA polymerase-beta chain ( $\mathrm{RpoC}$ ) and nonsense mutations in the nth excision repair gene. Single-nucleotide polymorphisms (SNPs) were associated with drug resistance. Besides that, genomic deletion mechanisms in Mycobacterium leprae-variable number tandem repeat (VNTR), called TTC repeats are described and might lead to microbiological nonresponse in treated cases of leprosy, which add a chapter to Mycobacterium leprae $[11,12]$.

Daily MCMC treatment was well tolerated. All patients were monitored in the laboratory, with no changes in blood count, liver, kidney, and pancreatic function. One patient complained of dizziness and vertigo within the first month of treatment, which subsequently disappeared.

With the MCMC treatment, all patients improved skin lesions, infiltrations, and reduced neural thickening, with improved function of the affected nerves (both visually and by palpation). There was a rapid reduction in $\mathrm{BI}$, higher than that usually described with the MDTMB treatment [13].

In addition to the bactericidal effect, all drugs used have recognized anti-inflammatory activity. This fact may have contributed to the control of LR.

Moxifloxacin has important immunomodulatory properties, such as the inhibition of TNF- $\alpha$ and IL-6, which are involved in the pathogenesis of the LR, especially type 2 , contributing to homeostasis in LL patients $[14,15]$.

Clarithromycin is a macrolide with known anti-inflammatory activity, with immunomodulatory effects on inflammatory cells, fibroblasts, and epithelial cells, in addition to modulating the expression of cytokines and chemokines [16].

Small trials have already tested the efficacy of moxifloxacin and clarithromycin in LL patients, both used separately and for short periods $[17,18]$.

Minocycline is a second-line drug in the treatment of leprosy. A robust trial demonstrated the effectiveness and tolerability for minocycline and clarithromycin longterm use in this disease. This drug is usefulness in cases of drug resistance also in the control of LR due to its immunomodulatory properties $[19,20]$.

Clofazimine is a first-line drug in the treatment of leprosy, which has the advantage of not inducing drug resistance known through molecular biology techniques, except in a few studies showing resistance in inoculation in mice. It is a option to control LR, especially type $2[21,22]$.

Interestingly, two patients who remained with a positive smear after MCMC treatment continued to express type 2 LR with the need to use drugs against leprosy reactions. These patients may have a longer period of disease evolution, between the beginning of the first symptoms and the beginning of treatment. A delay of fewer than 3 years between initial symptoms of leprosy and the start of treatment may lead to a significantly faster clearance of bacilli from the body [6].

It is also possible that these two patients are not yet cured. The relapse rate is not low and can increased to patients treated with MDT-MB 24 month fixed-dose in contrast to patients treated until smear-negative $[21,23]$.

Type $1 \mathrm{LR}$ has always been understood as a purely immunological phenomenon, however, its occurrence has already been demonstrated in the presence of bacillary multiplication due to the detection of Mycobacterium leprae-antigen 85 (Ag85) in lesions of type 1 LR. Ag85 is a $30 \mathrm{kDA}$ mycolyl transferase secreted during growth and multiplication of Mycobacterium leprae [24].

On the other hand, ENL after the removal of MDTMB is associated with disease' relapse $[25,26]$.

All the patients' household contacts of this case series are healthy, ruling out the possibility of reinfection.

\section{Limitations}

This study has some limitations. It was a small, retrospective case series at a single institution, and there are no validated tools to measure treatment response in NR-Lep.

\section{Conclusions}

A subset of leprosy patients with high initial BI remains a serious public health problem in countries that are hyperendemic for leprosy. They may not respond satisfactorily to MDT-MB 12-24-month fixed-dose. These patients have genetically determined energy contributing to the perpetuation of the transmission chain. We present 4 patients with NR-Lep who have been successfully treated with the daily MCMC scheme presented in this case series. Large studies are needed to understand the unsatisfactory response to MDT-MB in order to make better therapeutic alternatives available, especially considering that there are already bet- 
ter drugs that can be used in this neglected disease. However, the long time required for large prospective cohorts can greatly influence the quality of life of these patients.

\section{Funding}

Funding support to this publication sponsored by Alliance Against Leprosy Institute.

\section{Acknowledgements}

To Dr. Fred Nietto (PhD) for his help in translating this manuscript. There are no conflicts of interest in their disclosure.

\section{Authors' Contribution}

All authors contributed equally to the elaboration of this manuscript.

\section{References}

1. GETZ B (1958) Leprosy research in Norway, 1850-1900. Med Hist 2: 65-67.

2. Han XY, Aung FM, Choon SE, Werner B (2014) Analysis of the leprosy agents Mycobacterium leprae and Mycobacterium lepromatosis in four countries. Am J Clin Pathol 142: 524-532.

3. Schurr E, Alcaïs A, de Léséleuc L, Abel L (2006) Genetic predisposition to leprosy: A major gene reveals novel pathways of immunity to Mycobacterium leprae. Semin Immunol 18: 404-410.

4. Mushtaq S, Dogra N, Dogra D, Faizi N (2020) Trends and patterns of leprosy over a decade in a tertiary care hospital in Northern India: A retrospective analysis. Indian J Dermatol Venereol Leprol 86: 141-149.

5. MoHo (2016) Guidelines for Surveillance, Care and Elimination of Leprosy as a Public Health problem: Technical and operational manual. In: Diseases. SoHS-DoSol, BVS - Biblioteca Virtual em Saúde, Brazil 1-51.

6. Amenu A, Saunderson P, Desta K, Byass P (2000) The pattern of decline in bacillary index after 2 years of WHO recommended multiple drug therapy: The AMFES cohort. Lepr Rev 71: 332-337.

7. Nicchio MVC, Araujo S, Martins LC, Pinheiro AV, Pereira DC, et al. (2016) Spatial and temporal epidemiology of Mycobacterium leprae infection among leprosy patients and household contacts of an endemic region in Southeast Brazil. Acta Trop 163: 38-45.

8. da Conceição Oliveira Coelho Fabri A, Carvalho APM, Araujo S, Goulart LR, de Mattos AMM, et al. (2015) Antigen-specific assessment of the immunological status of various groups in a leprosy endemic region. BMC Infect Dis 15: 218.

9. Shen J, Yan L, Sun $P$ (2015) Clinical features of relapse after multidrug therapy for leprosy in China. Lepr Rev 86: 165-169.

10. (2018) Guidelines for the diagnosis, treatment and prevention of leprosy. World Health Organization, Regional Office for South-East Asia, New Delhi.

11. Benjak A, Avanzi C, Singh P, Loiseau C, Girma S, et al. (2018) Phylogenomics and antimicrobial resistance of the leprosy bacillus Mycobacterium leprae. Nat Commun 9: 352.
12. Reja AHH, De A, Patra PK, Biswas S, Duttagupta U, et al. (2018) Genomic reduction at TTC repeats in the bacterial genome of treated cases of Hansen's Disease: A possible survival mechanism of Mycobacterium leprae. Indian J Dermatol 63: 449-454.

13. Jesudasan K, Vijayakumaran $\mathrm{P}$, Manimozhi N, Rao PS, Samuel P (1996) Effectiveness of MDT in multibacillary leprosy. Int J Lepr Other Mycobact Dis 64: 128-132.

14. Dalhoff A, Shalit I (2003) Immunomodulatory effects of quinolones. Lancet Infect Dis 3: 359-371.

15. Sales-Marques C, Cardoso CC, Alvarado-Arnez LE, Illaramendi X, Sales AM, et al. (2017) Genetic polymorphisms of the IL6 and NOD2 genes are risk factors for inflammatory reactions in leprosy. PLoS Negl Trop Dis 11: e0005754.

16. Arikata $M$, Itoh $Y$, Shichinohe $S$, Nakayama $M$, Ishigaki $H$, et al. (2019) Efficacy of clarithromycin against H5N1 and $\mathrm{H} 7 \mathrm{~N} 9$ avian influenza a virus infection in cynomolgus monkeys. Antiviral Research 171: 104591.

17. Pardillo FEF, Burgos J, Fajardo TT, Dela Cruz E, Abalos RM, et al. (2008) Powerful bactericidal activity of moxifloxacin in human leprosy. Antimicrob Agents Chemother 52: 3113-3117.

18. Chan GP, Garcia-Ignacio BY, Chavez VE, Livelo JB, Jimenez CL, et al. (1994) Clinical trial of clarithromycin for lepromatous leprosy. Antimicrob Agents Chemother 38: 515-517.

19. Rea TH (2000) Trials of daily, long-term minocycline and rifampin or clarithromycin and rifampin in the treatment of borderline lepromatous and lepromatous leprosy. Int $\mathrm{J}$ Lepr Other Mycobact Dis 68: 129-135.

20. Narang T, Sawatkar GU, Kumaran MS, Dogra S (2015) Minocycline for recurrent and/or chronic erythema nodosum leprosum. JAMA Dermatol 151: 1026-1028.

21. Shetty VP, Wakade AV, Ghate S, Pai VV, Ganapati R, et al. (2003) Viability and drug susceptibility testing of M. leprae using mouse footpad in 37 relapse cases of leprosy. Int $\mathrm{J}$ Lepr Other Mycobact Dis 71: 210-217.

22. Roy K, Sil A, Das NK, Bandyopadhyay D (2015) Effectiveness and safety of clofazimine and pentoxifylline in type 2 lepra reaction: A double-blind, randomized, controlled study. Int J Dermatol 54: 1325-1332.

23. Gelber RH, Balagon VF, Cellona RV (2004) The relapse rate in $\mathrm{MB}$ leprosy patients treated with 2-years of WHOMDT is not low. Int $\mathrm{J}$ Lepr Other Mycobact Dis 72: 493-500.

24. Save MP, Dighe AR, Natrajan M, Shetty VP (2016) Association of viable Mycobacterium leprae with Type 1 reaction in leprosy. Lepr Rev 87: 78-92.

25. Guerrero-Guerrero MI, Muvdi-Arenas S, León-Franco $\mathrm{Cl}$ (2012) Relapses in multibacillary leprosy patients: A retrospective cohort of 11 years in Colombia. Lepr Rev 83: 247-260.

26. Brito Mde F, Ximenes RA, Gallo ME, Buhrer-Sekula S (2008) Association between leprosy reactions after treatment and bacterial load evaluated using anti PGL-I serology and bacilloscopy. Rev Soc Bras Med Trop 41: 67-72. 